OAI-PMH: http://www.indteca.com/ojs/index.php/Revista Scientific/oai

Artículo Original / Original Article

\title{
Visión Teórica Humanística Educativa de la Generación Z 3.0 en Tiempos Complejos
}

\author{
Autor: Carlos Liborio Camacho Quintero \\ Universidad de Los Andes, ULA \\ ccamacho@ula.ve; clcamachoq71@gmail.com \\ Mérida, Venezuela
}

\section{Resumen}

El propósito de esta investigación es generar desde una visión teórica humanística estrategias cognitivas para la construcción de conocimiento de la generación Z 3.0, donde el docente expresa que sus estudiantes son unos verdaderos nativos digitales y con la teoría de la mente alcanzar resultados cognoscentes. Desde el punto de vista metodológico, el estudio se ubica dentro del paradigma cualitativo en el cual se hace uso del método hermenéutico dialéctico. En relación con los informantes claves fueron cuatro (4) docentes adscritos al Instituto de Geografía, en cuanto a la técnica se empleó la entrevista, y como instrumento se aplicó la entrevista semi estructurada. Los datos se procesaron por medio de la categorización de la información, mientras que la fiabilidad de la investigación está dada por la triangulación de la misma, y con referencia a la técnica de análisis, se hizo con la revisión del material recopilado y codificación del mismo para su respectiva interpretación. La investigación se apoya en un constructo teórico basado en la teoría de sistemas, que intenta develar en la era digital los procesos tecnológicos y ambientes de aprendizajes flexibles en aulas virtuales, el autor concluye que los informantes destacan la necesidad de ser capacitados en su praxis pedagógica para transformar las competencias curriculares basadas en e-learning y b-learning que responden a estos desafíos de la globalización.

Palabras clave: conocimiento; humanismo; docente; aprendizaje. 


\title{
Humanistic Educational Theoretical Vision of the Generation Z 3.0 in Complex Times
}

\begin{abstract}
The purpose of this research is to generate from a theoretical humanistic vision cognitive strategies for building knowledge of the Z 3.0 generation, where the teacher says that her students are a true digital natives and the theory of mind to achieve cognizant results. From the methodological point of view, the study is located within the qualitative paradigm in which the dialectical hermeneutic method is used. Regarding key informants were four (4) assigned to the Institute of Geography professors in technical interview was used, and as the semi-structured interview instrument applied. Data were processed using the categorization of information, while the reliability of research is given by triangulation thereof, and with reference to the analysis technique, was made by reviewing the material collected and coding thereof for its respective interpretation. The research is based on a theoretical construct based on systems theory, which attempts to unveil technological processes and flexible learning environments in virtual classrooms in the digital age. The author concludes that informants highlight the need to be trained in their pedagogical practice. to transform the curricular competences based on e-learning and blearning that respond to these challenges of globalization.
\end{abstract}

Keywords: knowledge; humanism; teacher; learning.

Date Received: 06-02-2018

Date Acceptance: 02-05-2018 


\section{Introducción}

La globalización en la sociedad de las redes de información y comunicación del sistema educativo forma parte de la humanidad, las relaciones interpersonales se mueven en la virtualidad afectando directamente en la familia, comunidad, contexto educativo y profesional, el humanismo formar parte del acervo intelectual, fundamentado en el funcionalismo e instrumentalismo cultural y existencial, estamos en un mundo tecnológico, esta era planetaria es compleja y específicamente la educación que esta inmiscuida en redes sociales inteligentes

La era planetaria representa un todo de la condición humana y deriva en partes que se deben conocer y comprender desde un enfoque complejo; la humanidad global está en contantes cambios y maneras de ver el mundo, la globalización desde un pensamiento virtual está mediada por la innovación del internet, igualmente los medios de comunicación y el uso que constituye la generación nativa digital en la sociedad del conocimiento.

La evolución del mundo afecta las actividades humanas, existe mucha incertidumbre por la era científica tecnológica, porque impacta los procesos educativos, la capacidad cognitiva humana nos lleva a ser personas reflexivas y comunicativas por la innovación en las redes sociales que llevan un mensaje desafiante, la brecha tecnológica es cada día menos exigua por la conectividad digital que envuelve tanto al discente como docente en las aulas.

La modernidad da paso a la postmodernidad en la era tecnológica, donde se observan dispositivos inteligentes basados en memorias diminutas de gran capacidad usadas en celulares, cámaras y computadoras, no obstante, los seres humanos no van hacer desplazados por esta tecnología, ellos la producen, lo análogo es solo historia y punto de partida para los nuevos avances digitales producidos por esta generación llamada Z.

Para Iñaki (2017): el término generación Z, también conocido como centennials, ha emergido de una generación que se caracteriza por depender 
de la tecnología y vivir habituados a las interacciones en un mundo virtual, su dependencia a los dispositivos móviles, computador, tablas digitales, pizarra electrónica, interconectados a las redes y plataformas sociales, es decir, el argot que aborda este entorno virtual.

El entorno virtual de esta generación Z, incrementa el pensamiento creativo en el estudiante, estos nativos digitales para comunicarse utilizan entornos digitales, les gusta la sinergia y el trabajo colaborativo, la finalidad es romper el paradigma de la educación 1.0 tradicional, dar el salto cualitativo a la educación 2.0 constructivista hasta llegar a la educación 3.0 o conectivista que es la era digital que permita enfrentar los retos de la sociedad educativa moderna.

Los estudiantes nativos digitales en esta era tecnológica actúan semánticamente diferente a los profesores Inmigrantes digitales y análogos, es interesante mencionar que la generación $Z$, su cerebro es complejo y fisiológicamente disímil tanto a los inmigrantes digitales como a los análogos, como consecuencia de haber crecido en un mundo virtual y cibernético.

Esta brecha socio cognitiva generacional impacta el desarrollo de esta generación, la educación está pasando por una crisis económica suscitada en el mundo que afecta significativamente a la educación tradicional, por tal argumento la educación virtual es una posible solución en pregrado y postgrado, los docentes del siglo XXI debe modernizar su pensamiento, actualizar sus competencias para evitar instruir con contenidos del siglo XIX y siglo XX a estudiantes que manejan la tecnología y por ende están actualizados en cualquier materia.

La praxeología en el ejercicio docente a nivel universitario ha permitido evidenciar que la llegada de las TIC en el escenario educativo es necesaria para mejorar la calidad educativa, la web 2.0 o redes sociales generan tranquilidad pedagógica y pueden convertirse en sinergia para el desarrollo de propuestas educativas virtuales, asimismo la tecnología facilita el aprendizaje 
colaborativo y construcción de comunidades interconectadas donde todos somos aprendices.

Este hecho nos plantea como investigadores el reto de reconsiderar el docente nuevo basado en principios tecnológicos en la educación presencial y semipresencial, a fin de que éstas puedan acceder al conocimiento virtual desde una mirada propia, creativa, crítica, beneficiosa, optimizando en este camino su formación integral frente a las exigencias vanguardistas que imponen el dominio de las nuevas tecnologías de información y comunicación.

Por lo antes expuesto, se formula la siguiente interrogante: ¿Cómo explicar desde la teoría de la mente el conocimiento de la generación Z, en el marco humanístico de la complejidad?, para responder a la pregunta anterior, se hace necesario sistematizar la investigación a través de expectativas, a saber: ¿Cómo aprende esta generación?, ¿Es posible que su cerebro este programado para codificar y decodificar de manera diferente?, ¿Cuál es el impacto de las nuevas tecnologías sobre la base cognitiva de esta generación?, ¿El proceso de enseñanza como esta concedido en la actualidad será funcional para la generación Z, para la educación 3.0?., esbozando estas preguntas se llega al propósito general de la investigación que es generar una visión teórica fundamentada desde la teoría de la mente que permita diseñar estrategias cognitivas para la construcción de conocimiento de la generación Z 3.0 en el marco humanístico de la complejidad.

\section{Referentes Teóricos}

\subsection{Antecedentes de la Investigación}

En toda investigación científica, el investigador debe profundizar para conocer el estado del arte del conocimiento, con una compilación y síntesis de aquellos que presentan puntos coincidentes con la investigación, apoyado en las ideas principales para argumentar la información, cuyos conceptos primarios se consideran de gran valor. 
Fernández y Fernández (2016): realizaron una investigación titulada "Los docentes de la Generación Z y sus competencias digitales", señalando que la presencia de recursos tecnológicos en los centros y las altas capacidades de los alumnos de la Generación Tecnológica o Generación Z, no son suficientes para desarrollar en los alumnos la competencia digital. Los autores afirman que la clave fundamental viene determinada por las competencias tecnológicas y pedagógicas de los docentes. En su trabajo analizaron el nivel de competencias en TIC de los profesores de Primaria y Secundaria estableciendo un marco competencial de referencia adaptado al ámbito educativo español, la relación se centra en la relevancia de la formación del profesorado, desarrollando en ellos habilidades y destrezas particulares, que les permitan adquirir las competencias indispensables para desempeño en forma efectiva, centrándose en que el estudiante despierte el interés por materias indispensables para el desarrollo de la humanidad.

Por su parte, Jauam (2013): desde la Universidad Abierta Interamericana presenta una investigación titulada "Percepción de la Generación Z y la escuela en el siglo XXl" con el propósito de conocer qué siente y piensa dicho grupo etario, partiendo de sus percepciones y comportamientos, sus creencias sobre la adaptabilidad de la escuela con las nuevas tecnologías y los modos de enseñanza del sistema educativo en la actualidad, es decir, entre los principales hallazgos se encontraron discrepancias generacionales sustantivas entre docentes y alumnos asociadas a la desactualización del sistema educativo actual, aunado a la debilidad en competencias digitales presentes en sus profesores. Asimismo, se sostiene la tesis sobre el mundo cambiante y globalizado en que les ha tocado vivir a estos sujetos denominados generación $Z$, permeados de estímulos tecnológicos de toda índole. Estas observaciones dan cuenta del desfase existente entre los modos de enseñar y aprender de este tiempo y las características de los estudiantes de hoy, predispuestos a lo digital, a la 
inmediatez y los contenidos en línea como preferencia de búsqueda de la información.

\subsection{Contexto Teórico}

Es pertinente mencionar que el investigador puede encontrar discrepancias en las visiones y posturas entre inmigrantes digitales y nativos digitales que pertenecen a la Generación Z. Por tanto, su labor es unificar las posturas y crear una mirada integrada de la realidad encontrada para profundizar, igualmente también se han seleccionado como referentes teóricos los preceptos de la Teoría de la Mente y el Pensamiento Complejo, a los efectos de intentar diseñar estrategias cognitivas para la construcción social del conocimiento, entendiendo las capacidades mentalistas que estos chicos han desarrollados a través de los estímulos de la cultura digital y la necesidad, que desde la docencia, se requiere de hacer un ejercicio reflexivo y complejo para enfrentar estos desafíos.

\section{Marco Metodológico}

\subsection{Materiales y Métodos}

La investigación se efectúo en el Instituto de Geografía, Facultad de Ciencias Forestales de la Universidad de Los Andes (ULA), destinado a diagnosticar las causas de los profesores que laboran en esa institución. En cuanto al no usar la tecnología adecuada con los estudiantes nativos digitales pertenecientes a la generación $Z$, sin embargo se asume como estrategia operacional para concretar la investigación, el método cualitativo con tendencia hermenéutico, por cuanto se busca la descripción de ciertas prácticas conductuales de los profesores de esta institución señalada anteriormente, en un espacio institucional específico, cuyas relaciones están reguladas por normas que establecen obligaciones, derechos y costumbres.

Para Martínez (2006a): “...se genera un debate que trasciende al 
método, aquí se enmarca la discusión sobre el llamado círculo hermenéutico y sus discusiones metodológicas". (pág. 23). Es preciso mencionar la importancia de la hermenéutica en investigaciones sociales, debido a que permite la interpretación a profundidad, proporcionando significado a los hallazgos de los informantes.

En este orden de ideas, la hermenéutica incorpora en el texto el método dialéctico para el sujeto donde el reconocimiento da apertura al investigador discernir en cada párrafo, igualmente el enfoque cualitativo integra la dialéctica en la hermenéutica, el ser humano por naturaleza es crítico, discursivo e interpretativo en su medio ambiente, el razonamiento y el dialogo da certeza minimizando errores, es necesario que el investigador entienda la ecuanimidad de sus corrientes epistemológicas.

Martínez (2006b): propone un diseño cualitativo del proceso investigativo fundamentado en pasos secuenciales que llevan al investigador a enfocarse secuencialmente desde la selección del método hasta llegar a la teorización.

Figura 1. Proceso Cualitativo.

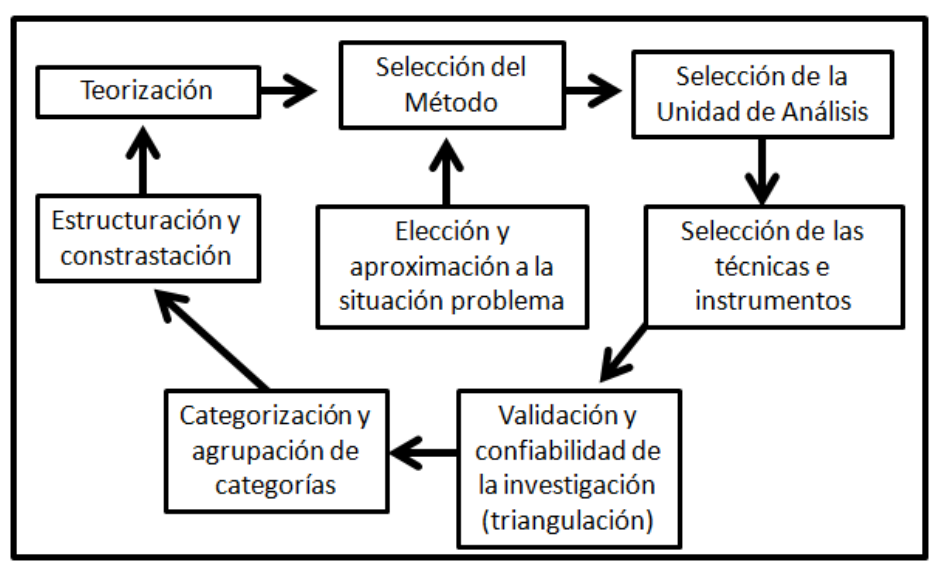

Fuente: Martínez (2006).

Al comenzar a ejecutar cada paso se inicia el proceso de recolección de los datos, la información suministrada por cada informante clave es fuente 
primordial al establecer el diagnóstico del contexto en estudio. Según Sabino (2002): “ ... un instrumento de recolección de datos es cualquier recurso de que se vale el investigador para acercarse a los fenómenos y extraer de ellos información" (pág. 99).

Sin embargo, la unidad de análisis detalla a cada informante clave en su vinculación directa o indirecta con el Instituto de Geografía, el proceso de categorización aunado a los objetivos específicos determina la delimitación de cada dimensión y hallazgo encontrado, el proyecto sigue encaminándose hasta diseñar la triangulación, que es de vital importancia para el desarrollo del constructo basado en la teorización y circulo de Dilthey.

Cuadro 1. Selección de las Unidades de Análisis (Informantes claves)

\begin{tabular}{|l|c|c|}
\hline \multirow{2}{*}{ Docentes del Instituto de Geografía ULA } & \multirow{2}{*}{ Cantidad } & Vinculación \\
\cline { 3 - 3 } & & Directa \\
\hline Sujeto IC . Docente Titular & 01 & $\mathrm{X}$ \\
\hline Sujeto IC 2 . Docente Asociado & 01 & $\mathrm{X}$ \\
\hline Sujeto IC 3. Docente Agregado & 01 & $\mathrm{X}$ \\
\hline Sujeto IC . Docente Instructor & 01 & $\mathrm{X}$ \\
\hline
\end{tabular}

Fuente: El Autor (2018).

En todo caso se asume el criterio de la muestra inicial intencional, en el sentido de consultar la opinión de aquellas personas que disponen de más información acerca del problema en estudio, enmarcado en las aristas de la educación 3.0, generación Z y el aprendizaje disruptivo. Esta fusión es tomada de las pesquisas de cada informante clave, generando la integración sistémica y socializada en el entorno virtual de esta generación Z. Es probable que algunas personas señalen un mayor impacto en su estabilidad emocional por ese problema en el ambiente de trabajo.

En este caso, los datos se recolectaron a través de las técnicas de 
entrevista y el procedimiento o método de observación participativa, mediante la aplicación de instrumentos como el diario de campo o guías que permitan cumplir con el objetivo de obtener información sobre los indicadores establecidos para el estudio de la educación 3.0, generación Z y el aprendizaje disruptivo.

La investigación de campo, con los datos recogidos constituyen al investigador piezas imprescindibles al analizar la guía de entrevista constituida por 22 preguntas abiertas para indagar sobre las competencias virtuales de los actores educativos (docentes) en su actuación pedagógica y de aprendizaje para la construcción del conocimiento, la población estuvo conformada por cuatro (04) docentes como informantes claves del Instituto mencionado, las respuestas que proporcionaron los informantes, forman el referencial el soporte y fundamente de cada objetivo planteado, esos axiomas sirven de basamento teórico para el estudio propuesto.

\subsection{Desarrollo, Resultados y Discusión}

El proceso de investigación secuencialmente abordó la información suministrada de las unidades de análisis por cada informante a través de la entrevista y la observación directa, así como el proceso de triangulación basado en integración de categorías, asimismo la posición de expertos permiten generar una visión teórica apoyada en la complejidad del profesor adscrito al Instituto, tomando en cuenta a que generación tecnológica pertenece cada informante clave, para corregir actitudes de resistencia al cambio, mejorar la comunicación con estos nativos digitales. 


\subsection{Categorización de las Experiencias}

Cuadro 2. Análisis del Informante Clave en Docencia Virtual.

SUBCATEGORÍA: Docencia Virtual

INFORMANTE CLAVE: Profesor Titular como Docente del Instituto de Geografía de la ULA $\left(\mathrm{IC}_{1}\right)$.

1. ¿Está el docente universitario dispuesto a renovar su pensamiento, métodos, medios, conocimientos, y todo el complexus necesario para el desarrollo de sus competencias digitales? Entrevistado: Le comento que mi trabajo consiste en dar clases de pregrado, posgrado y doctorado, imparto clases de manera presencial y reconozco que en pregrado existen muchos nativos digitales los cuales no se motivan en actividades tradicionales, soy de una generación conductista y análogo digital, lo cual siento una desventaja con estudiantes que les encanta las redes sociales, estoy de acuerdo con actualizar mis competencias.

2. ¿Conoce usted a la generación $Z$ y cree que se pueda trabajar grupal? Entrevistado: No he escuchado sobre esta generación, pero me imagino que son estudiantes que crecieron con tecnología, pienso que se puede trabajar grupal, la idea es romper paradigmas y dar el salto de la educación tradicional a la virtual.

3. ¿Se puede trabajar en la generación $Z$ con la comunicación lineal descendente para impartir instrucciones y exigir el cumplimiento de tareas virtuales? Entrevistado: Con gesto de disgusto comenta, en el Instituto hay docentes que presentan una comunicación lineal, la comunicación depende de su situación estudiantil ya que ellos interactúan con la tecnología. ¿Cree usted que el proceso de enseñanza en la generación Z 3.0 esta concedido en la educación actual? Entrevistado: Pienso que primero debemos formarnos tecnológicamente, ya que impartimos clases del siglo XX estando en contacto con estudiantes del siglo XXI que son totalmente nativos 
digitales. Cada uno puede contribuir a fin de concebir más agradable la interacción social y así evitar la resistencia tecnológica.

Fuente: El Autor (2018).

Figura 2. Triangulación de las Experiencias.

IC $\mathbf{C}_{1}$ : Profesor Titular como Docente del Instituto de Geografía de la ULA.

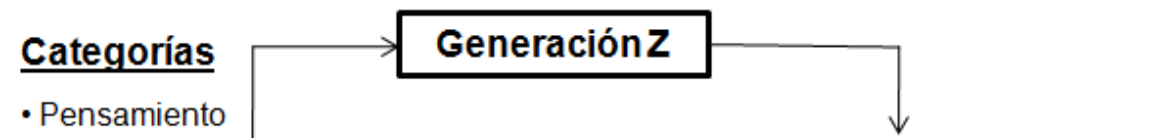

Lateral

•Pensamiento

Critico

- Globalización

Contexto

- Teoría de Universitario

Sistemas

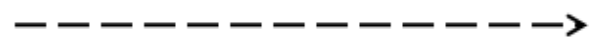

-Cognitivismo

- Conectividad
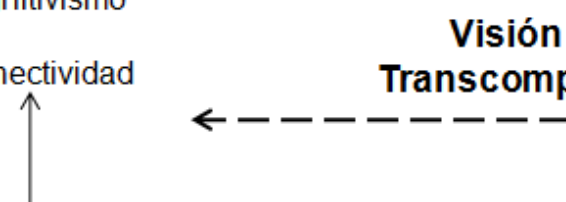

Visión

$\leftarrow------------$

Educación 3.0

$\stackrel{\infty}{\stackrel{m}{\circ}}$

destrezas humanas y técnicas desde una visión humanística y compleja al Aprendizajes Disruptivos

Fuente: El Autor (2018).

\section{Resultados}

En razón de los objetivos de la investigación, permitió caracterizar las Profesor del Instituto; los estudiantes sienten poco motivación debido a que 
los profesores de esta dependencia no utilizan las tecnologías, los profesores como inmigrantes digitales deben actualizarse en la educación virtual a causa que utilizan libros de data obsoleta, una solución es que el profesor trabaje con bibliografía de repositorios web actualizados, además esta discusión no permite socialización en la toma de decisiones estudiantiles con respecto a contenidos virtuales y clases en plataforma.

En consecuencia, muchos de los profesores son afectados por no recibir actualización en el área de las TIC, con incidencias en las materias de sistemas de información geográfica que requiere de conocimientos tecnológicos actualizados, ya que nos conseguimos con estudiantes nativos digitales que han hecho de la tecnología una forma de vida.

Según los informantes claves, el desconocimiento tecnológico se manifiesta en el instituto de diversas formas, a saber:

- Falta de motivación por no tener en sus cubículos tecnología obsoleta de cuarta y quinta generación.

- No existe posibilidad de actualizarse por la situación País.

- Problemas presentes por carencia de aprendizaje tecnológico ya que los profesores no saben utilizar la plataforma Moodle ULA para implementar cursos y clases e-learning lo cual motivaría al estudiante nativo digital. Martínez (2011): dentro del método hermenéutico dialectico, el autor considera pertinente plasmar los hallazgos apoyándose en el círculo hermenéutico sugerido por Dilthey, donde se encierra los análisis de la realidad a través del modelo de lo encontrado como producto del conocimiento y de la interpretación de las unidades de análisis. 
Figura 3. Circulo Hermenéutico de Dilthey.

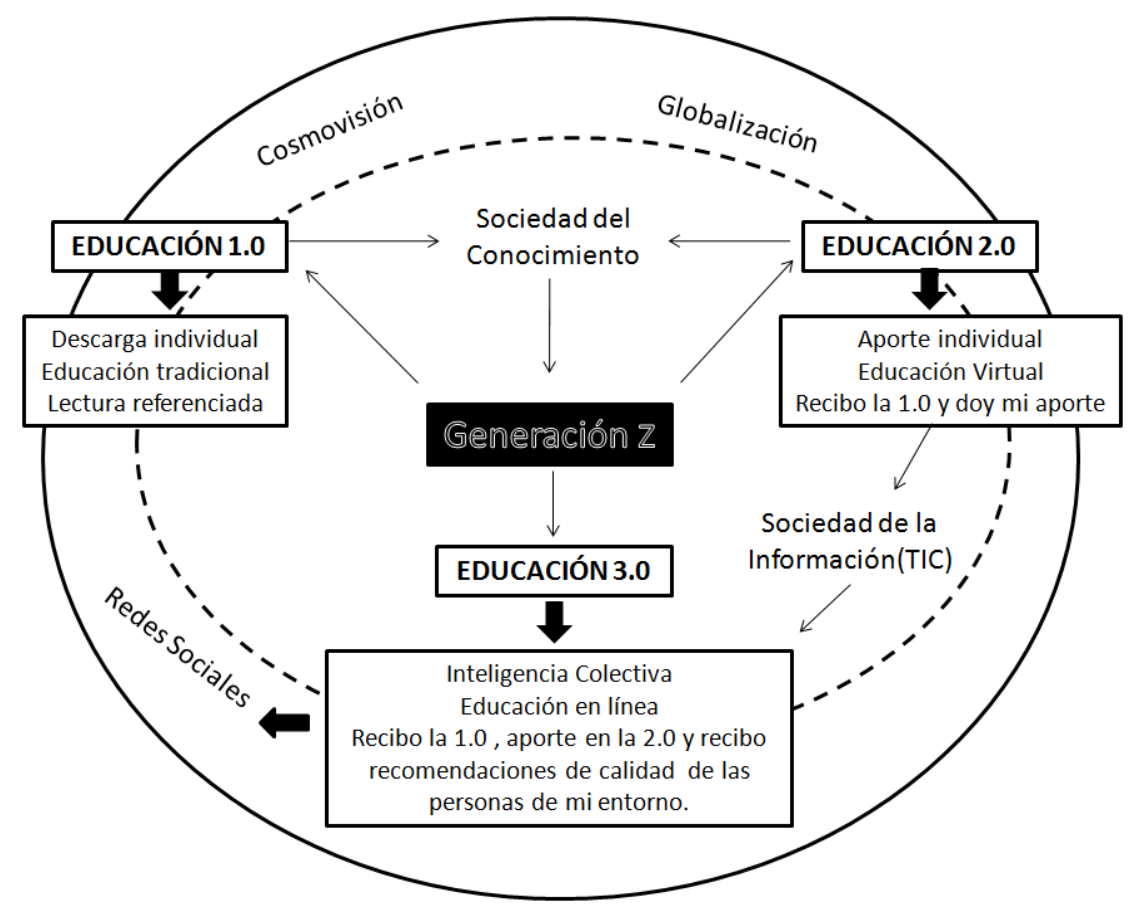

Fuente: El Autor (2018).

\section{Propuesta}

El circulo de Dilthey basado en el enfoque cualitativo, genera constructo teórico fundamentado las competencias del profesor del Instituto con respecto a la incidencia tecnológica, cabe destacar que se generó un constructo teórico basado en la generación $Z$ y aprendizajes disruptivos, el cual permitió la agrupación de teorías, hallazgos y dimensiones filosóficas para diseñar e identificar competencias e incluirlas en la unidad curricular, esta información cognoscitiva de los informantes clave entrevistados dilucido el constructo teórico basado en la teoría de sistemas.

La educación 3.0, rompe los paradigmas de profesores análogos e inmigrantes digitales bajo una visión compleja humanística, tal como se afirmó en esta investigación, se asocia a la Categorización y Triangulación que formo como resultado el circulo hermenéutico sugerido por Dilthey adaptado al 
Instituto de Geografía de la ULA; sistémicamente se crea el constructo teórico basado en la generación Z y aprendizaje disruptivo, el cual se representa así:

Figura 4. Estructura del Pensamiento Sistemático.

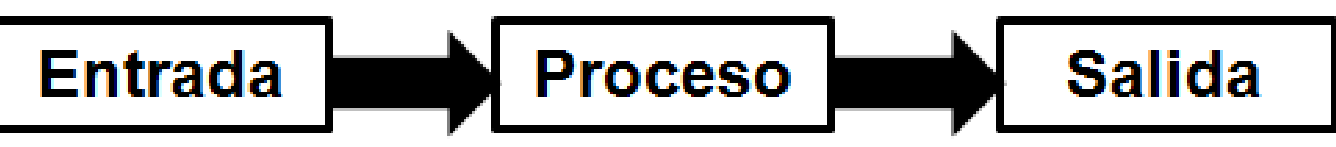

Fuente: El Autor (2018).

Entrada:

Informantes clave y el Instituto de Geografía de la ULA.

Proceso:

Generación Z y aprendizaje disruptivo, fusionado en las siguientes aristas:

- Flipped Classroom.

- Redes Sociales.

- E-learning y b-learning.

- Transformación holística curricular.

- Filtrado de información.

- Simulacro de la experiencia.

- Tecnología de Información y Comunicación (TIC).

- Retroalimentación.

Salida:

Constructo Teórico. 
Figura 5. Constructo Teórico enfocado a la Teoría de Sistemas.

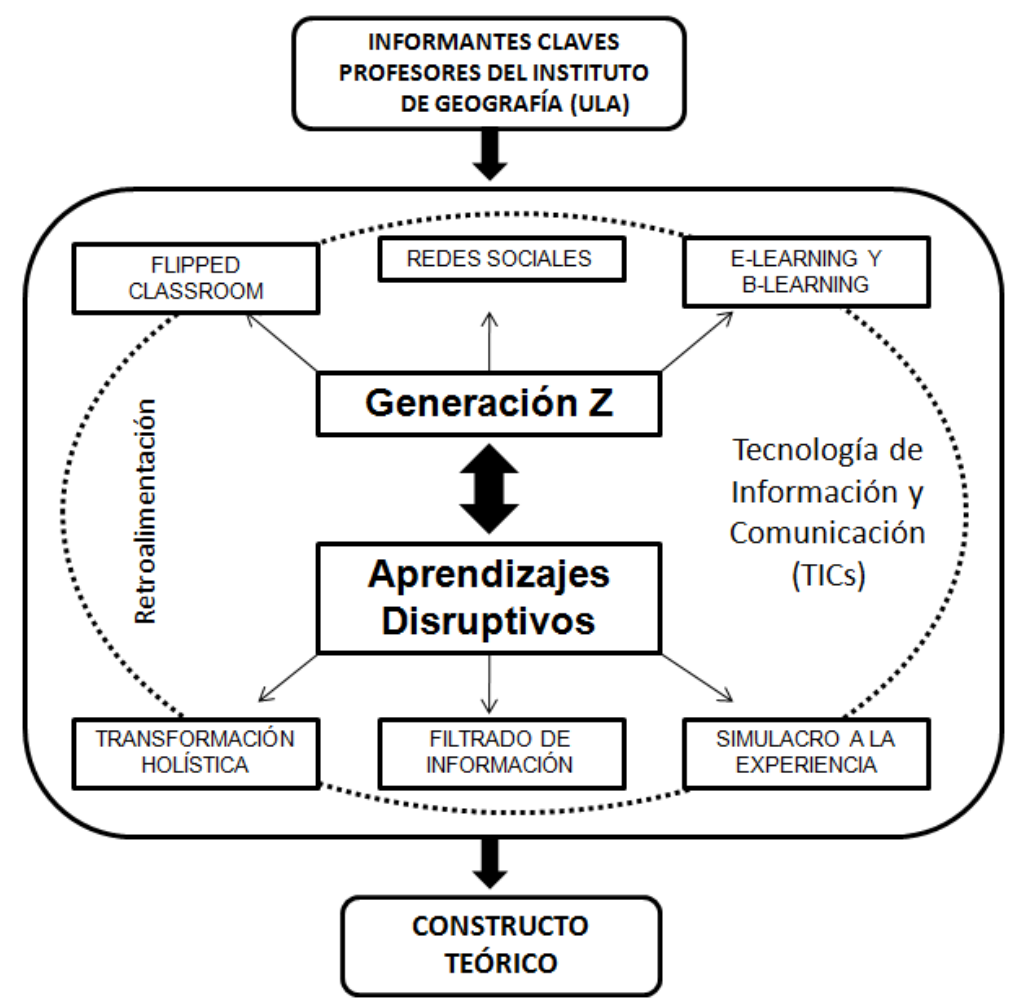

Fuente: El Autor (2018).

\subsection{Objetivos del Constructo Teórico}

- Incentivar al Profesor en el uso de las tecnologías.

- Capacitar al Profesor plataformas y simuladores web.

- Hacer uso de la pizarra digital cuando lo amerite.

- Diseño de programas instruccionales.

- Actualización curricular a la educación 3.0.

- Fomentar la motivación y reforzar la autoestima del profesor.

\section{Conclusiones}

Al caracterizar las funciones cerebrales de la generación $Z$, se debe manejar desde una perspectiva holística, esta investigación debe enmarcarse 
en la realidad circundante, con factores emergentes y antologías para buscar reflexiones y replanteamientos sociales que se adecuen a esos pensamientos complejos que piden ser abordados desde una perspectiva de la generación Z desde la teoría de la mente, en esta dinámica social circundante, es decir, que transcienda hasta cambiar actitudes de vida, con carácter creativo, autocrítico y proactivo.

Las estrategias cognitivas desde el enfoque humanístico para la construcción de conocimiento de la generación Z 3.0, genero aportes epistemológicos, metodológicos y prácticos de la investigación que son de gran utilidad para generar un modelo teórico interpretativo que facilite el entendimiento de esta generación, y abra el debate sobre una formación académica sólida para los profesores en el desarrollo de competencias digitales para la construcción del conocimiento de enseñanza aprendizaje, donde el estudio está determinado por la creatividad, la innovación y la tecnología, coadyuvando a generar constructos teóricos-tecnológicos en el campo de la educación.

Al teorizar los procesos explicativos de la generación Z 3.0, en razón de las implicaciones epistemológicas se enfoca en una visión hermenéutica dialéctica en tiempos complejos encauzado en educación virtual para activar a través de las creencias cibernéticas al ser humano reafirmando en él los valores, la ética, moral, responsabilidad, dignidad, integridad y excelencia para recuperar ese aprendizaje educativo que ha perdido tantos valores en la sociedad, los profesores deben actualizarse y al mismo tiempo enseñar virtualmente con plataformas educativas como la Moodle que es utilizada en la Universidad de Los Andes y así con la aplicación del constructo teórico la educación virtual presencial y semipresencial va hacer de gran ayuda en la formación del estudiante nacido en la generación $Z$. 


\section{Referencias}

Fernández, F., \& Fernández, M. (2016). Los docentes de la Generación Z y sus competencias digitales. Comunicar, XXIV(46), 97-105. ISSN digital: 1988-3293, ISSN impreso: 1134-3478. Recuperado de:

https://doi.org/10.3916/C46-2016-10

Jauam, G. (2013). "Percepciones de la Generación "Z" y la escuela en el siglo XXI". Rosario, Argentina: Universidad Abierta Interamericana. Sede Rosario - Campus Roca. Recuperado de:

http://imgbiblio.vaneduc.edu.ar/fulltext/files/TC113932.pdf

Martínez, M. (2006a,b). Ciencia y arte en la metodología cualitativa. México: Editorial Trillas, págs. 20-32.

Martínez, M. (2011). Epistemología y metodología cualitativa en las ciencias sociales. México: Editorial Trillas, págs. 30-55.

Sabino, C. (2002). El proceso de investigación. Venezuela: Editorial Panapo, págs. 92-99.

Iñaki, O. (2017). El Dilema de la Generación Z. Madrid, España: Deusto Business School. Este artículo se publicó originalmente en el diario ABC el día 16 de abril de 2017. Recuperado de:

http://www.inakiortega.com/2017/04/el-dilema-de-la-generacion-z.html 


\section{Carlos Liborio Camacho Quintero}

e-mail: ccamacho@ula.ve; clcamachoq71@gmail.com

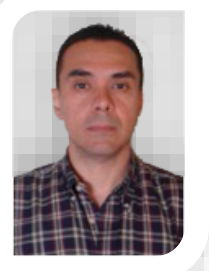

Nacido en Venezuela. Doctor en Gerencia Avanzada, Postdoctor en Gerencia para el Desarrollo Humano (ULA), Magíster Scientiarum en Gerencia Empresarial, Ingeniero de Sistemas, Licenciado en Administración de Empresas, Técnico Superior Universitario en Informática, Técnico Superior Universitario en Mercadotecnia. Administrador en TICs Instituto de Geografía, Facultad de Ciencias Forestales (ULA). Componente Docente (ULA), Docente en la Universidad Fermín Toro, Docente en la UPEL, Diplomado en Componente Docente en Educación a Distancia, Diplomado Internacional en TIC, Investigador PEII ONCTI, PEII ULA, cursos, talleres, congresos, ponencias, artículos científicos, tutor, jurado en defensas de tesis (ULA, UNEFA, UPEL y UFT).

El contenido de este manuscrito se difunde bajo una Licencia de Creative Commons Reconocimiento- 